\title{
Wherever is my arm? Impaired upper limb position accuracy in Complex Regional Pain Syndrome
}

Jennifer S. Lewis, Paula Kersten, Kathryn M. McPherson, Gordon J. Taylor, Nigel Harris, Candida S. McCabe, David R. Blake.

\section{INTRODUCTION}

"If the pain is very intense, even if I am touching something else I've got no idea where my arm exists" [26: Pg 115]. This description typifies the difficulty that those with CRPS express in knowing where their affected limb is positioned [26]. Knowledge of the position of one's limbs plays an essential role within the motor system - enabling accurate and smooth movements to be performed [12] and is a necessary component of daily functioning [17]. This sense involves a complex interaction of proprioceptive, vestibular, somatosensory and visual inputs from the periphery that interrelate with motor systems $[12,18,21]$. Interpretation of this multisensory information within the context of a centrally maintained representation of the limb or 'body schema' provides our fundamental sense of limb position $[20,21]$.

However, subjective perceptual disturbances of the affected limb are suggestive of distortions in body schema amongst those with CRPS. Features such as a desire to amputate $[7,9,26]$, perceptual distortions in size and shape $[26,31,32]$, lack of self-ownership $[11,13,26]$ and hostile feelings [26] have all been expressed by individuals about their affected limb. Cortical reorganisation in regions associated with the body schema (i.e. primary somatosensory cortex, posterior parietal lobe) have been revealed by brain imaging, providing further evidence of body schema disruption $[27,28,29,34]$.

Moreover, limb position sense is integral to motor performance yet motor dysfunction is well recognised in CRPS $[4,13,14,22,24,38,45,46,47,48]$. Brainimaging evidence of altered neural activity in motor cortices has also been found [29].

Despite acknowledged changes in body perception and motor function little is known about limb position sense and performance in CRPS. As such the aim of this study was to determine the degree to which objective experimental data supports the 'sense' or reported perception of limb positioning difficulty. We hypothesised that there was a statistically significant difference in affected upper limb position accuracy in those with CRPS when compared to healthy volunteers. In addition, we wished to determine whether there was a relationship between limb position accuracy and self-perception of the affected limb. Given that vision of the limb plays an important role in updating the body schema $[8,10]$ we aimed to establish the extent to which vision may contribute to limb positioning accuracy. By addressing these aspects we set out to provide insights into the mechanisms of limb position performance in CRPS and how this knowledge might inform clinical practice. 


\section{METHODS}

\section{Participants}

Twenty participants aged between 18 and 65 years who met the International Association of the Study of Pain classification criteria for CRPS Type I and II [43] of one upper limb were drawn from a UK population. Additional inclusion criteria were that they could perform $90^{\circ}$ shoulder abduction bilaterally, could verbally communicate and had no co-morbidity such as diabetic neuropathy that might influence performance. Those with severely impaired eyesight that could not be rectified with the use of visual aids were excluded. Participants were recruited from patients attending a national CRPS referral hospital. Twenty healthy volunteers with no history of chronic pain and who matched the patient's age and gender were also recruited. All participants gave written consent and data were collected in accordance with a protocol approved by the Local Research Ethics Committee and NHS Foundation Trust.

\section{Procedures}

Experimental study design

A controlled experimental design was used to determine the degree of upper limb position accuracy in patients with unilateral CRPS compared to healthy volunteers. Whilst seated in the centre of a quiet, windowless room, participants were asked to position one arm at a time in a series of horizontal positions corresponding to the hours of 9 o'clock through to 3 o'clock. The position of hours on a clock face in the horizontal plane was used as a cognitive internal construct because it comprised precise positions that were universally known. Prior to the experiment, clock hour positions were randomly selected by computer from a possible four for each arm (Right arm: 12, 1, 2, 3 o'clock Left arm: 9,10,11,12 o'clock). A set comprised performing three randomised positions with the same arm.

Coloured removable markers located on the dorsal aspects of both wrists were the points from which the participant's upper limb position was determined. Prompted by the researcher who stated a randomised hour, each participant moved their corresponding arm into a horizontal position at which they considered the wrist marker to be located at that hour. This procedure was repeated so that six sets were performed by each arm, hence every participant performed a total of 36 positions.

In order to establish the contribution of vision to limb position accuracy, the experiment was performed under two conditions: with the arm in view and with vision obscured. Opaque goggles were worn for the second condition. Participants undertook three sets of three positions with the left arm and three with the right in each condition. To minimise an order effect, half of each group commenced the experiment with the arm in view and half wearing goggles to obscure vision.

Data collection

A webcam was situated in the ceiling directly above the participant's chair and video captured an aerial view of each participant's arm positioning performance. The videos were digitally stored on a computer for later analysis. 


\section{Outcome measures}

Primary outcome measure

The primary outcome measure was the difference between target position (the hour) and limb position (determined by wrist marker) measured in degrees from the digital video using software [40]. In a trial of the experiment with healthy volunteers, the precision of this measure in limb position accuracy was shown to be within $1^{\circ}$

\section{Secondary outcomes}

1) Brief Pain Inventory (BPI) [6]

It was important to measure the extent of pain in order to describe and compare the CRPS study sample within the context of the general population. The BPI, a well validated pain measure was administered to determine a subjective rating of pain with higher scores denoting more severe pain. The participant completed the questionnaire prior to commencing the experiment.

The purpose of the following outcome assessments was to explore aspects of body perception amongst the two groups and to discover whether a relationship existed between these aspects and limb position accuracy.

2) Open question about limb position awareness

In order to capture the participant's perception of general awareness of limb position the following open question (as informed by a previous study [26]) was asked prior to the experiment;

"On a daily basis, how aware are you of the position of your limbs?" Answers were classified dichotomously and compared to the limb position performance data.

3) Mental image of upper limbs Asking participants to close their eyes, visualise and describe how their upper limbs appeared, captured a subjective mental representation of how individuals perceived both limbs. The researcher drew a line drawing to illustrate this description. The strength of this approach was that verbal descriptions of both arms were captured in illustrative form. Other nonpictorial descriptions were added in free text alongside the image. CRPS participants were asked to describe their unaffected limb first.

After opening their eyes, participants were asked to state whether the picture was an accurate representation of their mental image and had the opportunity to amend it accordingly. The resultant descriptions were then compared to the upper limb position accuracy findings.

\section{Effect size and group sample size calculation}

Given the lack of data in this area, we used a pragmatic approach to propose what would arguably be clinically relevant in terms of limb position recognition in normal functioning. A significant difference between the groups was estimated to be $10^{\circ}$ difference between the target and actual limb position. Limited data were available on which to estimate the standard deviation (SD). The expected range for participant's deviation from target position was $0-60^{\circ}$. Assuming a normally distributed response and six SDs across the range the SD was estimated at $10^{\circ}$. The estimated effect size was therefore 1. 
A sample size of 17 in each group was assessed to have $80 \%$ power to detect a difference in means of $10^{\circ}$ assuming that the common standard deviation was $10^{\circ}$ using an ANOVA with a 0.05 two-sided significance level. To account for the possibility of incomplete data, 20 participants were recruited for each group.

\section{Data analysis}

Analysis of primary outcome

Participants' videos were digitally analysed using computer software [40]. This programme enabled the researcher to draw digital lines on the video images. For the purposes of angle measurement, a standardised centre point across all videos was determined by the right hand edge of the vertical floor marker at $0^{\circ}$ and the horizontal shoulder position in each video. Still pictures for each arm clock position were created from the participant video and a line was digitally drawn from the centre point to the wrist marker for each position. From these lines, the programme could automatically calculate angles. The difference in degrees between the known clock hour angle and corresponding arm position angle was then calculated to determine the accuracy of each arm position that was performed (see figure 1). Given that the patient group had CRPS of a single arm, it was important to establish position accuracy of the affected limb separate from that of the unaffected limb. Hence, results from the study group are presented in this manner. However, since hand dominance was found to have no significant effect on position accuracy $(p=0.462)$ in healthy volunteers, data combining both arms are given for this group.

\section{(Position of figure 1 image, caption and key)}

\section{Statistical analysis}

A distribution plot showed that healthy volunteer limb position accuracy data was not normally distributed. Results are therefore reported as median values and interquartile ranges are given. Non-parametric analyses were performed using the Mann-Whitney $u$ test to compare between groups. A Bonferroni correction [1] determined a $p$ value of 0.008 or less as statistically significant for the between group comparisons. A $p$ value of 0.05 or less was used for within group comparisons as the variables were not independent. SPSS version 15 [42] was used to analyse data.

Analysis of secondary outcomes

Data from the BPI was statistically analysed using SPSS version 15 [42] Responses to the open question were classified in a dichotomous manner to either 'normal awareness' or 'difficulty in awareness'. If participants expressed any difficulty in knowing where their affected limbs were in response to the question it was categorised as 'difficulty in awareness'

No suitable method of scoring mental image representations was found in the literature. Given this novel approach a simple scoring system based on the principles of content rating was devised. Each drawing was rated on the presence of three aspects of distortion in mental representation. The rating given was 'no distortion', 'distortion' or 'severe distortion'. If either a distortion in size or shape was depicted within the drawing or the accompanying textual descriptions, i.e. that it was not anatomically consistent with the actual shape 
of the limb, the rating 'distortion' was given. If two or more segments of the body were missing this was rated as a 'severe distortion'.

\section{RESULTS}

Participant characteristics (table 1)

Twenty participants took part in each group. The CRPS and control groups were well matched for age and gender.

\section{(Position of Table 1)}

\section{Primary outcomes}

\section{a) Comparison of arm position accuracy between CRPS and healthy volunteer groups}

The error between the target clock position and the actual position in both experimental conditions combined was significantly greater in the CRPS group than the control group (table 2). Therefore the CRPS group was significantly less accurate in positioning of the affected limb only $(p<0.001)$, and both limbs combined than the control group $(p<0.001)$.

\section{(Position of table 2)}

b) Comparison between affected and unaffected arm position accuracy Within the CRPS group, no significant statistical difference in overall limb position accuracy (i.e. combined experimental condition data) between the affected and non-affected arms was found (table 3 ).

\section{(Position of table 3)}

c) Effect of vision on affected and unaffected arm position accuracy Comparisons of position accuracy in the two experimental visual conditions revealed that positioning of the affected limb was significantly more accurate when the limb was viewed compared to when it was not. Limb position accuracy of the unaffected arm between 'in view' and 'not in view' conditions was not significant (table 4).

\section{(Position of table 4)}

\section{Secondary outcomes}

\section{1) Brief Pain Inventory (BPI) results}

The CRPS group had moderate pain intensity and pain interference as measured by the BPI (table 5).

\section{(Position of table 5)}

2) General awareness of limb position

Sixty percent $(n=12)$ of those with CRPS reported some difficulty in general awareness of the position of their affected limb. Of these, six commented that they had to look at their affected limb in order to know where it was. Some stated that pain gave them an increased awareness of the limb but that this did not aid a sense of position. The control group reported no difficulty in limb position awareness. 
When comparing subjective reports and arm position accuracy within the CRPS group, no significant difference between the two variables was found (table 6). Therefore there was no association between subjective reports of limb position awareness and limb position accuracy.

\section{(Position of table 6)}

\section{3) Mental representation of upper limbs}

Within the CRPS group 95\% $(n=19)$ depicted a presence of one or more distortion such as a misshapen digit, an enlarged section of arm or were unable to visualise an anatomical part of the limb. Fifteen percent $(n=3)$ portrayed a severe distortion in mental representation where two or more segments of the limb were missing. Figure 2 illustrates an example of a severe mental representation as described by a 48-year-old female with right arm CRPS Type I of five year duration.

\section{(Position of figure 2)}

Two participants portrayed distortions in the mental representation of the unaffected arm. One participant with CRPS described no distortions in the mental representation of both upper limbs. The healthy volunteer group expressed no distortion in mental representation.

As data showed the presence of mental image distortions amongst those with CRPS, it was important to establish whether there was a direct relationship between the level of affected limb position accuracy and the extent of mental representation distortions by stratifying these aspects (table 7). From this exploratory data, no association was found between the degree of positioning accuracy and the extent of distortion within the CRPS group.

\section{(Position of table 7)}

In summary, those with CRPS were significantly less accurate in the positioning of both the affected and unaffected limbs when compared to healthy volunteers. Furthermore, they were significantly more accurate in positioning when their affected arm was in view compared to when it was not. Viewing the unaffected arm had no effect on position accuracy. Many had difficulty in general awareness of the position of their affected limb, although there was no association between that and limb position accuracy performance. The majority of those with CRPS depicted distortions in the mental representation of the affected limb and in some cases the unaffected limb.

\section{DISCUSSION}

This study has confirmed the hypothesis that those with upper limb CRPS have impaired limb positioning performance compared to healthy volunteers. Experimental data corroborate previous patient reports of a difficulty in affected limb position sense [26]. Findings revealed further important differences. These are discussed in the context of current scientific understanding and subsequent implications to clinical practice. 


\section{Bilateral impairment in a unilateral condition}

One may have expected that poor limb position accuracy would occur only in the CRPS affected limb. Pathophysiological features such as pain, vaso- and sudomotor changes, biomechanical restrictions due to muscle weakness and peripheral alterations in nociceptive processing could provide a plausible causative explanation. Yet, both the affected and unaffected limbs were found to be impaired in position accuracy, so alternative explanations are sought.

Perhaps these inaccuracies are a consequence of CRPS spreading into the unaffected limb such that early sub-clinical symptoms within this arm were responsible $[37,47]$. Although a reasonable explanation, in other studies only $4 \%$ [37] to $10 \%$ [47] of cases were found where spreading of symptomology into another limb occurred. Therefore, such an uncommon incidence is unlikely to account for the significant unaffected limb inaccuracy within this CRPS study sample.

Rather than peripheral pathology being the cause, perhaps a disruption in central processing is responsible. Growing evidence of alterations in the unaffected limb within the CRPS literature supports this view. Ribbers et al. [35] found that those with left hand CRPS also had motor impairments such as deficits in the execution of movements of the unaffected right hand.

Sensory disturbances including impaired pinprick and temperature sensations were found by Rommel et al. [36] in the unaffected limb ipsilateral to the CRPS affected limb in over a third of cases. Conversely, by using a mirror to create a visual illusion, CRPS symptoms have been generated on the affected side when only the unaffected side was touched [2]. Acerra and Moseley [2] showed that when patients with unilateral CRPS of the upper limb viewed a mirror image of their unaffected limb being stimulated in an area corresponding to an allodynic region of the affected side, patients experienced pain in that unstimulated allodynic region (termed dysynchiria) of the affected arm. In the absence of peripheral sensory input, this phenomenon can be explained by the brain generating perceived pain within the allodynic area in response to the visual perception that the painful area has been touched.

Taken together, these findings illustrate a variety of abnormal features occurring in a part of the body with no known CRPS pathology. Alterations in tactile, pain and temperature sensations, impairment in motor performance and limb position accuracy provide strong evidence that central mechanisms play a key role. Precisely where these alterations occur within the sensorimotor system remains unclear.

\section{Upper limb position accuracy and vision}

Findings revealed that affected limb position performance was significantly improved by viewing that arm. This experimental finding was borne out by participant reports of typically being reliant on visual cues to locate the affected limb and highlights the important role that vision plays in enhancing limb position accuracy. Given that vision made no difference in unaffected limb accuracy suggests that there is greater reliance on visual input to position the affected side. Hence, vision potentially plays a vital role in frequently updating the central representation of the affected limb to ascertain 
where it is in space [15]. Conceivably, body schema representation of the affected limb position is more transitory than that of the unaffected limb [15].

The use of visual manipulation has been shown to alter other pathologic features of CRPS. Mirror visual feedback of the unaffected limb resulted in a reduction in pain and swelling of the affected limb [30]. Sumitani et al. [44] found that adaptation to visual field displacement towards the unaffected side by $20^{\circ}$ with the use of prism spectacles could also alleviate pain in CRPS. Hence the use of vision within the therapeutic context can be key in ameliorating symptoms. In contrast, visual input may also evoke symptoms such as when generating dysynchiria as previously discussed [2].

Upper limb position and mental representation of the limb

A distorted mental representation of the affected limb was presented by all but one within the CRPS group thus confirming previous reports of perceived distortions of the affected limb [26,31,32]. Fifteen percent of the CRPS group had such a severely distorted mental representation that they were unable to visualise two or more segments of their affected arm. Two participants also depicted distortions of the unaffected side. As there was no known CRPS pathology of the unaffected side, these distortions could possibly be explained by altered central processing within cortical centres responsible for limb representation as demonstrated by brain imaging studies [25,27,28,34]. It is acknowledged that this unvalidated measure was devised specifically for this study hence these results are exploratory.

Central mechanisms: a feasible explanation

The following findings support the view that central mechanisms contribute to limb position impairment in CRPS:

1) Bilateral positioning impairment in a unilateral pain condition.

2) Vision significantly improved the ability to position the affected upper limb

3) Subjective mental images of the affected limb were distorted.

A disrupted body schema of the affected limb, which in turn impairs the ability to accurately position the limb, might be one such mechanism. Body perception disturbances, specifically distortions in mental image, as well as cortical remapping in body schema associated regions, are indicative of body schema disruption $[11,13,14,25,26,27,28,31,32,34]$. Consequently, an anatomically distorted affected limb schema would provide incorrect reference information for movement planning [33]. Hence limb position accuracy would be impaired.

This theory does not wholly explain why performance of the unaffected limb was also poor. Feasibly, spatial perceptual deficits exhibited by those with CRPS such that their subjective midline shifted towards the affected side [44] may alter internal spatial constructs for movement planning. Consequently 
performance accuracy in both limbs could be affected thus accounting for bilateral discrepancies.

Responsible for integrating visual, tactile and proprioceptive inputs and sensory-motor information, the post-parietal cortex may play a key role $[16,19$, 41]. Importantly, post-parietal lesions typically result in spatial deficits and clinical disturbances in body representation [39]. Although lesions are not known in CRPS, evidence of abnormal increased neural activation within both parietal lobes has been shown [29]. Furthermore, the degree of motor impairment correlated with activations of the parietal and motor cortices [29]. An alternative causative mechanism may therefore be that integration of multisensory and motor information about limb position within the parietal lobe is disrupted. Hence, limb position accuracy is affected as a consequence. Our research group have found considerable deficits in clinical tests associated with parietal lobe functioning in patients with CRPS. [Cohen $\mathrm{H}, \mathrm{McCabe} \mathrm{CS}$, Harris N, Blake DR. UNPUBLISHED DATA].

Two specific mechanisms have been proposed here although altered processing at spinal and mid brain levels may also serve to influence limb position accuracy.

Nonetheless, the contribution of pain within such a central mechanism is believed to be influential. A correlation between the amount of pain and degree of cortical reorganisation supports this view [28,34]. The extent to which pain may precipitate or perpetuate impairments in limb position accuracy remains unknown.

\section{Clinical implications}

Deficits in upper limb positioning have considerable relevance to daily functioning. For instance, inaccuracies could cause reaching and grasping misjudgements possibly resulting in accidents and injury. Objectively assessing patient's limb positioning ability within clinical practice is vital. Given that vision enhances positioning accuracy, treatment strategies involving visually concentrating on the limb during functional activities are advised.

A study limitation was that the experimental design had not been validated. However, alternative methods involving repeated measurements of single joint positioning rather than the whole limb were not functionally relevant $[3,5,8,10]$. Furthermore, due to the design of the experiment, the data is only relevant to those with upper limb CRPS.

In conclusion, findings have revealed that impairments in upper limb position accuracy are evident amongst those with CRPS adding further weight to the proposal of including motor dysfunction signs and symptoms in diagnostic criteria [23]. Bilateral impairment in a unilateral condition would suggest that central mechanisms might be responsible. Quite what the exact processes are remains unclear.

Further research is required to determine the incidence of limb positioning impairment amongst the wider CRPS and pathologic pain populations. 
Studies regarding the relationship with pain and the impact of such impairments on function are also necessary.

\section{Acknowledgements}

This study was supported by grants from the Department of Health, Arthritis Research Campaign and The Royal National Hospital for Rheumatic Diseases donated funds. Particular thanks goes to the participants for their immense contribution to this study. We declare no conflict of interest.

\section{REFERENCES}

[1] Abdi, H. Bonferroni and Sidak corrections for multiple comparisons. In: Salkind NJ, editor. Encyclopaedia of Measurement and Statistics. Thousand Oaks. Sage 2007;103-107.

[2] Acerra N, Moseley GL. Dysynchiria: Watching the mirror image of the unaffected limb elicits pain on the affected side. Neurology 2005;65:751-753.

[3] Bearne LM, Coomer AF, Hurley MV. Upper limb sensorimotor function and functional performance in patients with rheumatoid arthritis. Disabil Rehabil 2007;29:13:1035-1039.

[4] Bruehl S, Harden RN, Galer BS, Saltz S, Backonja M, Stanton-Hicks M. Complex regional pain syndrome: are there distinct subtypes and sequential stages of the syndrome? Pain 2002;95:1-2: 119-124.

[5] Bullock-Saxton JE, Wong WJ, Hogan N. The influence of age on weightbearing joint reposition sense of the knee. Exp Brain Res 2001;136:3:400-406

[6] Cleeland CS, Ryan KM. Pain assessment: global use of the Brief Pain Inventory. Ann Acad Med 1994; 23:129-138

[7] Dielissen P, Claassen AT, Veldman P, Goris, R. Amputation for reflex sympathetic dystrophy. J Bone Joint Surg 1995;77-B:270-273.

[8] Dover G, Powers M., Reliability of joint position sense and force reproduction measures during internal and external rotation of the shoulder. $J$ Athl Train 2003;38:304-310.

[9] Dudzinski D. "Amputate my arm please-I don't want it anymore". J Clin Ethics 2005;16:196-201.

[10] Ferrell WR, Crighton A, Sturrock RD., Position Sense at the Proximal Interphalangeal Joint Is Distorted in Patients with Rheumatoid-Arthritis of Finger Joints. Exp Physiol 1992;77:675-680.

[11] Förderreuther S, Sailer U, Straube, A. Impaired self-perception of the hand in complex regional pain syndrome (CRPS). Pain 2004;110:756-761. 
[12] Frith CD, Blakemore SJ, Wolpert DM. Abnormalities in the awareness and control of action. Philos.Trans.R.Soc.Lond B Biol.Sci 2000;355:17711788.

[13] Galer BS, Butler S, Jensen MP. Case-Reports and Hypothesis - A Neglect-Like Syndrome May be Responsible for the Motor Disturbance in Reflex Sympathetic Dystrophy (Complex-Regional-Pain-Syndrome-1). J Pain Symptom Manage 1995;10:385-391.

[14] Galer BS, Jensen M. Neglect-like symptoms in complex regional pain syndrome: Results of a self-administered survey. J Pain Symptom Manage 1999;18:213-217.

[15] Ghez C, Gordon J, Ghilardi M. Impairments of reaching movements in patients without proprioception. II Effects of visual information on accuracy. J Neurophysiol 1995;73:361-372.

[16] Goodale M, Milner D. Sight unseen. Oxford. Oxford University Press 2004

[17] Gordon J, Ghilardi M, Ghez C., Impairments of reaching movements in patients without proprioception. I Spatial errors. J Neurophysiol 1995;73:347360.

[18] Graziano MSA. Where is my arm? The relative role of vision and proprioception in the neuronal representation of limb position. Proc Natl Acad Sci U S A 1999;96:10418-10421.

[19] Graziano MSA, Botvinick MM. How the brain represents the body: insights from neurophysiology and psychology. In: Prinz W, Hommel B editors. Common Mechanisms in Perception and Action. Oxford. Oxford University Press 2002;19:136-157.

[20] Haggard P, Taylor-Clarke M, Kennett S. Tactile perception, cortical representation and the bodily self. Curr Biol 2003;13:R170-R173.

[21] Haggard P, Wolpert DM. Disorders of body scheme. In: Leiguarda R editor. Higher-order motor disorders: from neuroanatomy and neurobiology to clinical neurology.. Oxford. Oxford University Press 2005:261-271.

[22] Harden RN, Bruehl S, Galer BS, Saltz S, Bertram M, Backonja M, Gayles R, Rudin N, Bhugra MK, Stanton-Hicks M. Complex regional pain syndrome: are the IASP diagnostic criteria valid and sufficiently comprehensive? Pain 1999; 83:211-219.

[23] Harden RN, Bruehl S. Diagnostic criteria: The statistical derivation of the four criterion factors. In: Wilson P, Stanton-Hicks M, Harden RN, editors. CRPS: Current diagnosis and therapy. Seattle. IASP Press 2005;32:45-58.

[24] Jänig W, Baron R. Complex Regional Pain Syndrome: mystery explained? Lancet Neurol 2003;30:687-697. 
[25] Juottonen K, Gockel M, Silen T, Hurri H, Hari R, Forss N. Altered central sensorimotor processing in patients with complex regional pain syndrome. Pain 2002;98:315-323.

[26] Lewis JS, Kersten P, McCabe CS, McPherson KM, Blake DR. Body perception disturbance: A contribution to pain in Complex Regional Pain Syndrome. Pain 2007;133:111-119.

[27] Maihöfner C, Handwerker HO, Neundorfer B, Birklein F. Patterns of cortical reorganization in complex regional pain syndrome. Neurology 2003;61:1707-1715.

[28] Maihöfner C, Handwerker HO, Neundorfer B, Birklein F. Cortical reorganization during recovery from complex regional pain syndrome. Neurology 2004;63:693-701.

[29] Maihöfner C, Baron R, Decol R, Binder A, Birklein F, Deuschl G, Handwerker $\mathrm{HO}$, Schattschneider J. The motor system shows adaptive changes in complex regional pain syndrome. Brain 2007;130:2671-2687.

[30] McCabe CS, Haigh RC, Ring EFJ, Halligan PW, Wall PD, Blake DR. A controlled pilot study of the utility of mirror visual feedback in the treatment of complex regional pain syndrome (type 1). Rheumatology 2003;42:97-101.

[31] McCabe CS, Shenker N, Lewis J, Blake DR., Impaired self-perception of the hand in complex regional pain syndrome (CRPS) 2005 Pain [S.Förderreuther,U. Sailer, A. Staube, Pain 2004; 1007:56-761].

[32] Moseley L. Distorted body image in complex regional pain syndrome. Neurology 2005;65:773.

[33] Overney L, Michel CM, Harris IM, Pegna AJ. Cerebral processes in mental transformations of body parts: Recognition prior to rotation. Cogn Brain Res 2005;25: 722-734.

[34] Pleger B, Tegenthoff $M$, Ragert $P$, Forster A, Dinse H, Schwenkreis $P$, Nicolas $\mathrm{V}$, Maier $\mathrm{C}$. Sensorimotor returning in complex regional pain syndrome parallels pain reduction. Ann Neurol 2005; 57:425-429.

[35] Ribbers GM, Mulder T, Geurts AC, den Otter RA. Reflex sympathetic dystrophy of the left hand and motor impairments of the unaffected right hand: Impaired central motor processing? Arch Phys Med Rehabil 2002;83:81-85.

[36] Rommel, O, Gehling M, Dertwinkel R, Witscher K, Zenz M, Malin JP, W. Hemisensory impairment in patients with complex regional pain syndrome. Pain 1999;80:95-101.

[37] Sandroni P, Benrud-Larson L, McClelland R, Low P. Complex regional pain syndrome type I: incidence and prevalence in Olmsted county, a population-based study. Pain 2003;103:199-207. 
[38] Schwartzman R J, Kerrigan J. The Movement Disorder of Reflex Sympathetic Dystrophy. Neurology 1990;40:57-61.

[39] Schwoebel J, Coslett HB. Evidence for multiple, distinct representations of the human body. J Cogn Neurosci. 2005;17:543-553.

[40] Siliconcoach. Siliconcoach student. Version 6.1.4.1. 2004. www.siliconcoach.com.

[41] Sirigu A, Daprati E, Ciancia S, Giraux P, Nighoghossian N, Posada A, Haggard P., Altered awareness of voluntary action after damage to the parietal cortex, Nat Neurosci 2004;7:80-84.

[42] SPSS. SPSS for windows. [15.0] 2006.

[43] Stanton-Hicks M, Jänig W, Hassenbusch S, Haddox JD, Boas R, Wilson $\mathrm{P}$, Reflex sympathetic dystrophy: Changing concepts and taxonomy. Pain 1995;63:127-133

[44] Sumitani M, Rossetti Y, Shibata M, Matsuda Y, Sakaue G, Inoue T, Mashimo T, Miyauchi S. Prism adaptation to optical deviation alleviates pathologic pain. Neurology 2007;68:128-133.

[45] van Hilten JJ, Van de Beek WJT, Vein AA, van Dijk JG, Middelkoop HAM. Clinical aspects of multifocal or generalized tonic dystonia in reflex sympathetic dystrophy. Neurology 2001;56:1762-1765.

[46] van Rijn MA, Marinus J, Putter $\mathrm{H}$, van Hilten JJ. Onset and progression of dystonia in Complex Regional Pain Syndrome. Pain 2007;130:287-293.

[47] Veldman P, Reynen H, Arntz I, Goris R. Signs and symptoms of reflex sympathetic dystrophy: prospective study of 829 patients. Lancet 1993; 342:1012-1016.

[48] Wasner G, Schattschneider J, Binder A, Baron R. Complex regional pain syndrome - diagnostic, mechanisms, CNS involvement and therapy. Spinal Cord 2003;41:61-75. 